\title{
Split $(n+t)$-color partitions and Gordon-McIntosh eight order mock theta functions
}

\author{
A.K. Agarwal* \\ Centre for Advanced Study in Mathematics \\ Panjab University \\ Chandigarh-160014, India \\ aka@pu.ac.in \\ G. Sood ${ }^{\dagger}$ \\ Centre for Advanced Study in Mathematics \\ Panjab University \\ Chandigarh-160014, India \\ garimasood18@gmail.com
}

Submitted: Sep 19, 2013; Accepted: Jun 3, 2014; Published: Jun 9, 2014

Mathematics Subject Classifications: 05A15, 05A17, 11P81

\begin{abstract}
In 2004, the first author gave the combinatorial interpretations of four mock theta functions of Srinivasa Ramanujan using $n$-color partitions which were introduced by himself and G.E. Andrews in 1987. In this paper we introduce a new class of partitions and call them "split $(n+t)$-color partitions". These new partitions generalize Agarwal-Andrews $(n+t)$-color partitions. We use these new combinatorial objects and give combinatorial meaning to two basic functions of Gordon-McIntosh found in 2000. They used these functions to establish the modular transformation formulas for certain eight order mock theta functions. The work done here has great potential for further research.
\end{abstract}

Keywords: Mock theta fuctions, $(n+t)$-color partitions, split $(n+t)$-color partitions, combinatorial interpretations.

${ }^{*}$ Emeritus Scientist, CSIR.

${ }^{\dagger}$ Supported by CSIR Research Grant No. 09/135(0636)/2011-EMR-I. 


\section{Introduction}

Srinivasa Ramanujan is regarded as the most influential Indian mathematician of the twentieth century. The remarkable thing about him was: without any formal training and in the midst of extreme poverty he was able to do original mathematics research of the highest quality. He did not give up even in the face of his death. In his last letter dated 12 January, 1920, about three months before his death, to G.H. Hardy he listed 17 functions which he called mock theta functions. He separated these 17 functions into three classes. First containing 4 functions of order 3, second containing 10 functions of order 5 and the third containing 3 functions of order 7 . Watson [17] found three more functions of order 3 and two more of order 5 appear in the lost notebook [15]. Mock theta functions of order 2, 6, 8 and 10 have also been studied in [13], [6], [10] and [8], respectively. Further results for mock theta functions of even order are given in [14]. For the definition of mock theta functions, the reader is referred to [16]. Ramanujan did not give a precise definition of "order". A definition of the "order" of a mock theta function was given by Gordon and McIntosh in [11].

Mock theta functions have been studied in different directions. For example, Ramanujan divided mock theta functions of order 5 into two Groups A and B and gave many identities, without proofs, satisfied by mock theta functions in each group. Watson [18] proved all of Ramanujan's identities by the methods of rearrangement of series. He also made use of the basic hypergeometric series for finding new representations of many mock theta functions. The partition theory has also been used in the study of mock theta functions. A partition of a positive integer $n$ is any non-increasing sequence of positive integers whose sum is $n$. 0 also has a partition called "empty partition". The rank of a partition is defined to be the largest part minus the number of its parts. Partition theoretic interpretations of some of the mock theta functions are found in the literature. For example, $\chi(q)$, defined by (1.1) below, has been interpreted as generating function for partitions into odd parts without gaps (cf. [9]). Very recently Bringmann and Ono [7] redefined mock theta functions as the holomorphic projection of weight $1 / 2$ weak maass forms and used their ideas in solving the classical problem of obtaining formulas for $N_{e}(n)$ (resp. $N_{0}(n)$ ), the number of partitions of $n$ with even (resp. odd) rank by showing the equivalence of this problem and the problem of deriving exact formulas for the coefficients $\alpha(n)$ of the series

$$
f(q)=1+\sum_{n=1}^{\infty} \alpha(n) q^{n}=\sum_{n=0}^{\infty} \frac{q^{n^{2}}}{\left(-q ; q^{2}\right)_{n}},
$$

where $f(q)$ is the first mock theta function of order 3 in Ramanujan's list of 17 mock theta functions (cf. [9]) and

$$
(a ; q)_{n}=\prod_{i=0}^{\infty} \frac{\left(1-a q^{i}\right)}{\left(1-a q^{n+i}\right)}
$$

for any constant $a$. 
Recently in [1], the first author interpreted the following four mock theta functions (first is of order 3 and the remaining three are of order 5) of S. Ramanujan:

$$
\begin{gathered}
\chi(q)=\sum_{m=1}^{\infty} \frac{q^{m^{2}}}{\left(q ; q^{2}\right)_{m}}, \\
F_{0}(q)=\sum_{m=0}^{\infty} \frac{q^{2 m^{2}}}{\left(q ; q^{2}\right)_{m}}, \\
\phi_{0}(q)=\sum_{m=0}^{\infty} q^{m^{2}}\left(-q ; q^{2}\right)_{m}
\end{gathered}
$$

and

$$
\phi_{1}(q)=\sum_{m=0}^{\infty} q^{(m+1)^{2}}\left(-q ; q^{2}\right)_{m},
$$

combinatorially by using $n$-color partitions.

First we recall the definitions of a partition with " $(n+t)$-copies of $n$ " or " $(n+t)$-color partition" from [4] and that of the weighted difference from [3].

Definition 1.1. A partition with " $(n+t)$ copies of $n$ ", (also called an $(n+t)$-color partition), $t \geqslant 0$, is a partition in which a part of size $n, n \geqslant 0$, can occur in $n+t$ different colors denoted by subscripts: $n_{1}, n_{2}, \ldots, n_{n+t}$.

Thus for example, the partitions of 2 with " $(n+1)$ copies of $n$ " are

$$
\begin{array}{lll}
2_{1}, & 2_{1}+0_{1}, 1_{1}+1_{1}, 1_{1}+1_{1}+0_{1}, \\
2_{2}, & 2_{2}+0_{1}, 1_{2}+1_{1}, 1_{2}+1_{1}+0_{1} \\
2_{3}, & 2_{3}+0_{1}, 1_{2}+1_{2}, 1_{2}+1_{2}+0_{1} .
\end{array}
$$

Note that zeros are permitted if and only if $t$ is greater than or equal to one.

Definition 1.2 The weighted difference of two elements $m_{i}$ and $n_{j}, m \geqslant n$, is defined by $m-n-i-j$ and is denoted by $\left(\left(m_{i}-n_{j}\right)\right)$.

It was proved in [1] that the mock theta functions (1.1)-(1.4) have their $n$-color partition theoretic interpretations in the following theorems, respectively,

Theorem 1: For $\nu \geqslant 1$, let $A_{1}(\nu)$ denote the number of $n$-color partitions of $\nu$ such that the parts and their subscripts have the same parity, the smallest part is $k_{k}$ for some $k$, and the weighted difference of any two consecutive parts is 0 . Then,

$$
\sum_{\nu=1}^{\infty} A_{1}(\nu) q^{\nu}=\chi(q)
$$


Theorem 2: For $\nu \geqslant 0$, let $A_{2}(\nu)$ denote the number of $n$-color partitions of $\nu$ such that the parts and their subscripts have the same parity, the subscripts are greater than 1 , the smallest part is $k_{k}$ for some $k$, and the weighted difference of any two consecutive parts is 0 . Then,

$$
\sum_{\nu=0}^{\infty} A_{2}(\nu) q^{\nu}=F_{0}(q) .
$$

Theorem 3: For $\nu \geqslant 0$, let $A_{3}(\nu)$ denote the number of $n$-color partitions of $\nu$ such that only the first copy of the odd parts and the second copy of the even parts are used, that is, the parts are of the type $(2 k-1)_{1}$ or $(2 k)_{2}$, the minimum part is $1_{1}$ or $2_{2}$, and the weighted difference of any two consecutive parts is 0 . Then,

$$
\sum_{\nu=0}^{\infty} A_{3}(\nu) q^{\nu}=\phi_{0}(q)
$$

Theorem 4: For $\nu \geqslant 1$, let $A_{4}(\nu)$ denote the number of $n$-color partitions of $\nu$ such that only the first copy of the odd parts and the second copy of the even parts are used, the minimum part is $1_{1}$, and the weighted difference of any two consecutive parts is 0 . Then,

$$
\sum_{\nu=1}^{\infty} A_{4}(\nu) q^{\nu}=\phi_{1}(q) .
$$

One more mock theta function of order 5 given by

$$
F_{1}(q)=\sum_{n=0}^{\infty} \frac{q^{2 n(n+1)}}{\left(q ; q^{2}\right)_{n+1}},
$$

was also interpreted combinatorially in [5] using partitions with " $n+2$ copies of $n$ " or " $(n+2)$-color partitions" as follows:

Theorem 5: For $\nu \geqslant 0$, let $A_{5}(\nu)$ denote the number of $(n+2)$-color partitions of $\nu$ such that the parts and their subscripts have the same parity, the subscripts are greater than 1 , for some $i, i_{i+2}$ is a part and the weighted difference of any two consecutive parts is 0 . Then,

$$
\sum_{\nu=0}^{\infty} A_{5}(\nu) q^{\nu}=F_{1}(q) .
$$


Remark: An extensive survey of mock theta functions is given in [12].

In our next section, we introduce a new class of partitions and call them "split $(n+t)$-color partitions". These new partitions generalize the $(n+t)$-color partitions defined above. We use these partitions in interpreting combinatorially the following two Gordon-McIntosh basic functions from [10]:

$$
V_{0}(q)=1+2 \sum_{n=1}^{\infty} \frac{q^{n^{2}}\left(-q ; q^{2}\right)_{n}}{\left(q ; q^{2}\right)_{n}}
$$

and

$$
V_{1}(q)=\sum_{n=1}^{\infty} \frac{q^{n^{2}}\left(-q ; q^{2}\right)_{n-1}}{\left(q ; q^{2}\right)_{n}} .
$$

Remark: These functions $V_{0}$ and $V_{1}$ were used by Gordon and McIntosh to establish the modular transformation formulas for the following eight order mock theta functions:

$$
\begin{aligned}
& S_{0}(q)=\sum_{n=0}^{\infty} \frac{q^{n^{2}}\left(-q ; q^{2}\right)_{n}}{\left(-q^{2} ; q^{2}\right)_{n}} \\
& S_{1}(q)=\sum_{n=0}^{\infty} \frac{q^{n(n+2)}\left(-q ; q^{2}\right)_{n}}{\left(-q^{2} ; q^{2}\right)_{n}}, \\
& T_{0}(q)=\sum_{n=0}^{\infty} \frac{q^{(n+1)(n+2)}\left(-q^{2} ; q^{2}\right)_{n}}{\left(-q ; q^{2}\right)_{n+1}} \\
& T_{1}(q)=\sum_{n=0}^{\infty} \frac{q^{n(n+1)}\left(-q^{2} ; q^{2}\right)_{n}}{\left(-q ; q^{2}\right)_{n+1}} .
\end{aligned}
$$

We shall prove our main results in Section 3 and conclude in our last section with a direction for future work.

\section{Split $(n+t)$-color partitions and the main results}

Definition 2.1. Let $m_{i}$ be a part in an $(n+t)$-color partition of a non negative integer $\nu$. We split the color ' $i$ ' into two parts - 'the green part' and 'the red part' and denote them by ' $g$ ' and ' $r$ ', respectively, such that $1 \leqslant g \leqslant i, 0 \leqslant r \leqslant i-1$ and $i=g+r$. An $(n+t)$-color partition in which each part is split in this manner is called a split $(n+t)$-color partition.

Example. In $7_{3+2}$, the green part is 3 and the red part is 2 .

Remark. If the red part is 0 , we will not write it. Thus, for example, we will write $7_{5}$ for $7_{5+0}$. 
Remark. In this paper we will use only split $n$-color partition.

In our next section we shall prove that $V_{0}(q)$ and $V_{1}(q)$ have their combinatorial interpretations in the following theorems:

Theorem 2.1. For $\nu \geqslant 1$, let $A_{1}(\nu)$ denote the number of split $n$-color partitions of $\nu$ such that

(i) the parts and their subscripts have the same parity,

(ii) the red part of the subscripts cannot exceed 1 ,

(iii) the least part is either $k_{k}(k \geqslant 1)$ or $k_{(k-1)+1}(k \geqslant 2)$, and

(iv) the weighted difference of any two consecutive parts is 0.

Then

$$
V_{0}(q)=1+2 \sum_{\nu=1}^{\infty} A_{1}(\nu) q^{\nu} .
$$

Remark: In conditions (i) and (iv) we consider the whole subscript $i$, not its parts $g$ and $r$, separately.

Example. $A_{1}(5)=4$, the relevant partitions are $5_{5}, 5_{4+1}, 4_{2}+1_{1}, 4_{1+1}+1_{1}$.

Theorem 2.2. For $\nu \geqslant 1$, let $A_{2}(\nu)$ denote the number of split $n$-color partitions of $\nu$ such that

(i) the parts and their subscripts have the same parity,

(ii) the red part of the subscripts cannot exceed 1 ,

(iii) the least part is $k_{k}(k \geqslant 1)$, and

(iv) the weighted difference of any two consecutive parts is 0 .

Then

$$
V_{1}(q)=\sum_{\nu=1}^{\infty} A_{2}(\nu) q^{\nu} .
$$

Remark: As in Theorem 2.1, here also, in conditions (i) and (iv) we consider the whole subscript $i$, not its parts $g$ and $r$, separately.

Example. $A_{2}(5)=3$, the relevant partitions are $5_{5}, 4_{2}+1_{1}, 4_{1+1}+1_{1}$.

In the proofs given in the next section we use the notation $A_{i}(m, \nu)(1 \leqslant i \leqslant 2)$ to denote the number of split $n$-color partitions enumerated by $A_{i}(\nu)$ into $m$ parts with $A_{1}(0,0)=1$. Also, we shall write

$$
f_{1}(z, q)=\sum_{n=0}^{\infty} \frac{q^{n^{2}}\left(-q ; q^{2}\right)_{n} z^{n}}{\left(q ; q^{2}\right)_{n}}
$$


and

$$
f_{2}(z, q)=\sum_{n=1}^{\infty} \frac{q^{n^{2}}\left(-q ; q^{2}\right)_{n-1} z^{n}}{\left(q ; q^{2}\right)_{n}}
$$

where $|q|<1,|z|<|q|^{-1}$.

\section{Proofs}

\section{Proof of Theorem 2.1.}

We split the partitions enumerated by $A_{1}(m, \nu)$ into three classes:

(i) those that contain $1_{1}$ as a part,

(ii) those that contain $2_{1+1}$ as a part, and

(iii) those that contain $k_{k}(k>1)$ or $k_{(k-1)+1}(k>2)$ as a part.

We transform the partitions in class (i) by deleting $1_{1}$ and then subtracting 2 from all the remaining parts ignoring the subscripts. The transformed partition will be of the type enumerated by $A_{1}(m-1, \nu-2 m+1)$.

Next, we transform the partitions in class (ii) by deleting the part $2_{1+1}$ and then subtracting 4 from all the remaining parts ignoring the subscripts. The transformed partition will be of the type enumerated by $A_{1}(m-1, \nu-4 m+2)$.

Finally, we transform the partitions in class (iii) by replacing $k_{k}$ by $(k-1)_{(k-1)}$ or by replacing $k_{(k-1)+1}$ by $(k-1)_{(k-2)+1}$, as the case may be, and then subtracting 2 from all the remaining parts. The transformed partition will be of the type enumerated by $A_{1}(m, \nu-2 m+1)$. Clearly, the above transformations are reversible and so they establish a bijection between the partitions enumerated by $A_{1}(m, \nu)$ and those enumerated by $A_{1}(m-1, \nu-2 m+1)+A_{1}(m-1, \nu-4 m+2)+A_{1}(m, \nu-2 m+1)$. This leads to the identity

$$
A_{1}(m, \nu)=A_{1}(m-1, \nu-2 m+1)+A_{1}(m-1, \nu-4 m+2)+A_{1}(m, \nu-2 m+1) .
$$

Let

$$
h_{1}(z, q)=\sum_{\nu=0}^{\infty} \sum_{m=0}^{\infty} A_{1}(m, \nu) z^{m} q^{\nu}
$$

where $|q|<1,|z|<|q|^{-1}$.

Substituting for $A_{1}(m, \nu)$ from (3.1) into (3.2) and then simplifying we arrive at the $q$-functional equation

$$
h_{1}(z, q)=z q h_{1}\left(z q^{2}, q\right)+z q^{2} h_{1}\left(z q^{4}, q\right)+\frac{1}{q} h_{1}\left(z q^{2}, q\right) .
$$


Setting

$$
h_{1}(z, q)=\sum_{n=0}^{\infty} \alpha_{n}(q) z^{n}, \quad \alpha_{0}(q)=1,
$$

we may easily check by coefficient comparison in (3.3) that

$$
\alpha_{n}(q)=\frac{q^{n^{2}}\left(-q ; q^{2}\right)_{n}}{\left(q ; q^{2}\right)_{n}} .
$$

Thus

$$
h_{1}(z, q)=\sum_{n=0}^{\infty} \frac{q^{n^{2}}\left(-q ; q^{2}\right)_{n} z^{n}}{\left(q ; q^{2}\right)_{n}}=f_{1}(z, q) .
$$

Now

$$
\begin{aligned}
1+\sum_{\nu=1}^{\infty} A_{1}(\nu) q^{\nu} & =\sum_{\nu=0}^{\infty}\left(\sum_{m=0}^{\infty} A_{1}(m, \nu)\right) q^{\nu} \\
& =h_{1}(1, q) \\
& =f_{1}(1, q) \\
& =\sum_{n=0}^{\infty} \frac{q^{n^{2}}\left(-q ; q^{2}\right)_{n}}{\left(q ; q^{2}\right)_{n}} \\
& =1+\sum_{n=1}^{\infty} \frac{q^{n^{2}}\left(-q ; q^{2}\right)_{n}}{\left(q ; q^{2}\right)_{n}} .
\end{aligned}
$$

Using the penultimate equation in (1.11), we arrive at (2.1). This completes the proof of Theorem (2.1).

\section{Proof of Theorem 2.2.}

We split the partitions enumerated by $A_{2}(m, \nu)$ into three classes:

(i) those that contain $1_{1}$ as a part,

(ii) those that contain $k_{k}(k>1)$ as a part.

It is easy to see that there are $A_{1}(m-1, \nu-2 m+1)$ in class (i) and $\frac{1}{2}\left(A_{1}(m-1, \nu-4 m+2)+A_{1}(m, \nu-2 m+1)\right)$ in class (ii), so we get the identity

$$
A_{2}(m, \nu)=A_{1}(m-1, \nu-2 m+1)+\frac{1}{2}\left(A_{1}(m-1, \nu-4 m+2)+A_{1}(m, \nu-2 m+1)\right) .
$$

Let

$$
h_{2}(z, q)=\sum_{\nu=1}^{\infty} \sum_{m=0}^{\infty} A_{2}(m, \nu) z^{m} q^{\nu} .
$$

Translating (3.7) into a $q$-functional equation, we get

$$
h_{2}(z, q)=z q h_{1}\left(z q^{2}, q\right)+\frac{1}{2}\left(z q^{2} h_{1}\left(z q^{4}, q\right)+\frac{1}{q} h_{1}\left(z q^{2}, q\right)\right) .
$$


Setting

$$
h_{2}(z, q)=\sum_{n=1}^{\infty} \beta_{n}(q) z^{n},
$$

and using (3.4), we may easily check by coefficient comparison in (3.9) that

$$
\beta_{n}(q)=q^{2 n-1} \alpha_{n-1}(q)+\frac{1}{2}\left(q^{4 n-2} \alpha_{n-1}(q)+q^{2 n-1} \alpha_{n}(q)\right) .
$$

Substituting for $\alpha_{n}(q)$ from (3.5) into (3.11), we get

$$
\beta_{n}(q)=\frac{q^{n^{2}}\left(-q ; q^{2}\right)_{n-1}}{\left(q ; q^{2}\right)_{n}} .
$$

Thus

$$
h_{2}(z, q)=\sum_{n=1}^{\infty} \frac{q^{n^{2}}\left(-q ; q^{2}\right)_{n-1} z^{n}}{\left(q ; q^{2}\right)_{n}}=f_{2}(z, q) .
$$

Hence

$$
\begin{aligned}
\sum_{\nu=1}^{\infty} A_{2}(\nu) q^{\nu} & =\sum_{\nu=1}^{\infty}\left(\sum_{m=0}^{\infty} A_{2}(m, \nu)\right) q^{\nu} \\
& =h_{2}(1, q) \\
& =f_{2}(1, q) \\
& =\sum_{n=1}^{\infty} \frac{q^{n^{2}}\left(-q ; q^{2}\right)_{n-1}}{\left(q ; q^{2}\right)_{n}} \\
& =V_{1}(q) .
\end{aligned}
$$

This proves Theorem (2.2).

\section{Conclusion}

We hope that many more basic series can be interpreted combinatorially by using the split $(n+t)$-color partitions. Here we have given only two examples to illustrate the possibilities. It would be of interest if Rogers-Ramanujan type identities for split $(n+t)$ color partitions can be found as we have for $n$-color partitions in [2].

\section{References}

[1] A.K. Agarwal, n-color partition theoretic interpretations of some mock theta functions, Electron. J. Combin. 11(1), (2004), \#N14.

[2] A.K. Agarwal, Rogers-Ramanujan identities for $n$-color partitions, J. Number Theory 28(1988), 299-305. 
[3] A.K. Agarwal, and G.E. Andrews, Rogers-Ramanujan identities for partitions with " $N$ copies of $N$ ", J. Combin. Theory Ser.A, 45(1), (1987), 40-49.

[4] A.K. Agarwal, G.E. Andrews and D.M. Bressoud, The Bailey Lattice, J. Indian Math. Soc., 51(1987), 57-73.

[5] A.K. Agarwal and M. Rana, Two new combinatorial interpretations of a fifth order mock theta function, The Indian Mathematical Society, Special Centenary Volume, 1907-2007, (2008), 11-24.

[6] G.E. Andrews and D. Hickerson, Ramanujan's "Lost" notebook VII: The sixth order mock theta functions, Adv. Math. 89(1991), 60-105.

[7] K. Bringmann and K. Ono, The $f(q)$ mock theta function conjecture and partition ranks, Invent. Math. 165(2006), 243-266.

[8] Y.S. Choi, Tenth order mock theta functions in Ramanujan's lost notebook, Invent. Math. 136(1999), 497-596.

[9] N.J. Fine, Basic Hypergeometric Series and Applications, Mathematical Surveys and Monographs, No. 27, AMS(1988).

[10] B. Gordon and R.J. McIntosh, Some eight order mock theta functions, J. London Math. Soc., (2)62, (2000), 321-335.

[11] B. Gordon and R.J. McIntosh, Modular transformations of Ramanujan's fifth and seventh order mock theta functions, Ramanujan J. 7(2003), 193-222.

[12] B. Gordon and R.J. McIntosh, A survey of classical mock theta functions, Partitions, $q$-series, and modular forms, Dev. Math., 23, Springer, New York,(2012), 95-144.

[13] R.J. McIntosh, Second order mock theta functions, Canad. Math. Bulletin 50(2) (2007), 284-290.

[14] R.J. McIntosh, The $H$ and $K$ Family of Mock Theta Functions, Canad. J. Math. 64(2012), no.4, 935-960.

[15] S. Ramanujan, The "Lost" notebook and other unpublished papers, Narosa Publishing House, New Delhi (1988).

[16] P.V. Seshu Aiyar, G.H. Hardy and B.M. Wilson, Collected papers of Srinivasa Ramanujan, Cambridge Univ. Press (1927).

[17] G.N. Watson, The final problem: an account of the mock theta functions, J. London Math. Soc., 11(1936), 55-80.

[18] G.N. Watson, The mock theta functions (2), Proc. London Math. Soc., 42(1937), 274-304. 